\title{
Red hair and anesthetic requirement
}

\author{
Daniel I. Sessler, MD
}

Received: 12 January 2015/Accepted: 15 January 2015/Published online: 30 January 2015

(c) Canadian Anesthesiologists' Society 2015

Black, brown, and blond hair results from varying amounts of melanin, a black pigment. In contrast, red hair is caused by a mutation of the melanocortin-1 receptor. ${ }^{1-3}$ People with this mutation produce pheomelanin, which is yellow-red and accounts for the red hair and fair complexion consequent to defective melanocortin receptors.

There have long been rumours in the anesthesia community that procedures in patients with natural red hair can be challenging. Consistent with these admittedly vague impressions, investigators in several exploratory studies have concluded that redheads differ from people with other hair colours in their sensitivity to volatile anesthetics, local anesthetics, and opioids.

For example, Liem et al. concluded that redheaded women require $19 \%$ more desflurane than women with dark hair to prevent movement in response to supramaximal electrical stimulation. ${ }^{4}$ Melanocortin-1 receptor gene $(M C 1 R)$ knock-out rats also require a slightly greater minimum alveolar concentration (MAC) - a measure of anesthetic sensitivity based on movement in response to supramaximal pain ${ }^{5}$ - than wild-type rats. ${ }^{6}$ In mice, $M C 1 R$ mutations mediate kappa-opioid analgesia, but only in female mice. ${ }^{7}$ In subsequent studies, the same investigators found that $M C 1 R$ mutations in both mice and humans reduce pain sensitivity and increase analgesic responsiveness to $\mu$-receptor opioids. ${ }^{8}$ They also observed that the effect of this mutation is sex-specific in mice. ${ }^{9}$ In contrast, red hair does not influence propofol pharmacodynamics. ${ }^{10}$

D. I. Sessler, MD ( $₫)$

Michael Cudahy Professor and Chair, Department of Outcomes

Research, Cleveland Clinic, 9500 Euclid Ave - P77,

Cleveland, OH 44195, USA

e-mail: DS@OR.org

URL: http://www.OR.org
Although melanocortin-1 receptors have been identified in human pituitary tissue, glial cells, and cells of the human periaqueductal gray matter, ${ }^{11,12}$ the central nervous system is not a major site of $M C 1 R$ expression. ${ }^{13}$ Furthermore, MAC is probably largely mediated by the spinal cord ${ }^{14,15}$ rather than by higher centres. ${ }^{15,16}$ Nevertheless, there is considerable interaction among the various melanocortin receptors ${ }^{17,18}$ as all are stimulated by melanocortin, $\alpha$-melanocortin stimulating hormone (MSH), $\beta$-MSH, $\gamma$-MSH, and adrenocorticotropic hormone (ACTH). Some of these receptors are abundant in the central nervous system (CNS) and have similar affinities for $\alpha$-MSH and ACTH. ${ }^{13}$ A functional antagonism between the opioid and melanocortin systems has been suggested, as the receptors are co-localized throughout the $\mathrm{CNS}$, including in the locus ceruleus where their regulatory activities oppose each other. $^{19}$

Redheads are also more sensitive to thermal pain and resistant to the analgesic effects of subcutaneous lidocaine. ${ }^{20}$ Consistent with these experimental results, a subsequent cross-sectional study found that participants with $M C 1 R$ gene variants reported significantly more dental care-related anxiety and fear of dental pain than participants with no $M C 1 R$ gene variants. Subjects with the mutation were also more than twice as likely to avoid dental care as participants without $M C 1 R$ gene variants, even after controlling for general trait anxiety and sex. ${ }^{21}$

In this issue of the Journal, Gradwohl et al. ${ }^{22}$ describe a secondary analysis of patients drawn from a previously published study of anesthetic awareness in patients at high risk for recall. ${ }^{23}$ In their current analysis, the investigators compared four anesthetic characteristics in 319 self-reported redheads and 1,595 propensity-matched patients with other hair colours.

The first outcome the investigators considered was awareness with recall. Even in the high-risk patients they 
enrolled, only about three of 319 redheads would have been expected to have awareness with recall, and only two did. Gradwohl et al. thus have no statistical power for drawing any conclusion about awareness from these data.

Fortunately, Gradwohl et al. are much better positioned to consider anesthetic management. Their results show, with a reasonable degree of confidence, that volatile anesthetics, anesthetic adjuvants, and opioid administration were managed similarly in both redheads and patients with other hair colours. As the authors note, their results indicate that, at the very least, anesthesiologists in the two participating centres did not manage redheads differently from other patients.

Gradwohl et al. do not confuse the amount of anesthesia given with anesthetic requirement, and it remains possible that requirement by some measure would in fact have differed in the redheads. This distinction is important because the previous rodent and human studies evaluated highly specific measures of anesthetic requirement, such as MAC and pain sensitivity, in optimal non-clinical contexts. The difficulty, of course, is that anesthesia includes unconsciousness, immobility, control of autonomic responses, and amnesia. There is thus no unitary measure of "anesthesia", although the bispectral index (BIS) of the electroencephalogram is probably the best-accepted single overall measure and has been used to guide automatic propofol and remifentanil delivery. ${ }^{24,25}$

Gradwohl et al. thus used the relationship between volatile anesthetic partial pressure (age-adjusted MAC) and BIS as a measure of anesthetic sensitivity, a technique we have used in other contexts. ${ }^{26}$ The analysis was adjusted for opioid and anesthetic adjuvant administration, along with some patient and surgical characteristics. There were no clinically important differences in anesthetic sensitivity between redheads and patients with other hair colours. Among the outcomes tested by Gradwohl et al., the relationship between MAC and BIS is probably the most sensitive for potential effects of red hair on anesthesia. They also evaluated duration of postanesthesia recovery and nausea and vomiting. Neither would have been expected to vary much, and neither did.

The question, then, is why the results of Gradwohl et al. differ from a half-dozen rodent and exploratory human studies. The most obvious explanation is that previous studies evaluated specific outcomes, including MAC and pain sensitivity, under optimal circumstances. Such subtle effects may simply be undetectable even in a careful clinical cohort study. Nevertheless, it is also hardly unusual that many consistent small studies are proven wrong by a subsequent large trial. ${ }^{27}$ Aside from the fact that small trials are statistically fragile and thus are simply wrong more often than generally thought, ${ }^{28}$ methodologic details sometimes provide an explanation.
Gradwohl et al. ${ }^{22}$ restricted enrolment to patients who self-reported natural red hair. In this respect, their study differs from the studies of Liem et al. and Binkley et al. Liem et al. restricted enrolment to volunteers with bright red hair and conducted both biochemical tests on hair and genetic analysis on their volunteers. Binkley et al. conducted genetic analysis on their subjects. ${ }^{21}$ To the extent that Gradwohl et al. included patients who did not truly have natural red hair (and the predisposing $M C 1 R$ mutation), it would diminish apparent differences with their control patients.

The study of Gradwohl et al. was a prospective cohort rather than a randomized controlled trial since it is hardly possible to randomize hair colour. As a consequence, the investigators had neither the protections against selection bias and confounding provided by randomization nor the protection against measurement bias provided by blinding. Thus, the key to the validity of their results is the statistical analysis which was well done using appropriate techniques.

In summary, Gradwohl et al. ${ }^{22}$ evaluated various measures of anesthetic sensitivity in a prospective cohort of patients. They found that redheads and a matched group of control patients were given similar amounts of volatile anesthetics, opioids, and anesthetic adjuvants. Furthermore, there was a similar relationship between MAC and BIS in redheads and in patients with other hair colours. Their results suggest that previously reported relatively subtle differences in sensitivity to volatile anesthetics, local anesthetics, and opioids may not extend to the general population of self-reported redheads.

\section{Les roux et les anesthésiques}

La couleur noire, brune ou blonde des cheveux est le résultat de quantités variables d'un pigment noir, la mélanine. Les cheveux et poils roux diffèrent des autres couleurs, car cette couleur est provoquée par une mutation $\mathrm{du}$ récepteur de la mélanocortine de type $1 .^{1-3}$ Les personnes porteuses de cette mutation produisent de la phéomélanine dont la couleur jaune-rouge est responsable de la rousseur des cheveux et du teint pâle associés aux récepteurs défectifs de la mélanocortine.

Des rumeurs courent depuis longtemps dans la communauté anesthésiologique selon lesquelles les personnes naturellement rousses peuvent être des patients problématiques. Tout en reconnaissant le caractère vague de ces impressions, plusieurs études exploratoires ont semblé les conforter en concluant que les roux diffèrent des personnes ayant une autre couleur de cheveux sur le plan de leur sensibilité aux anesthésiques volatils, aux anesthésiques locaux, et aux opioïdes. 
Liem et coll. ont, par exemple, conclu que des femmes rousses nécessitent $19 \%$ de plus de desflurane que les femmes ayant des cheveux noirs pour la prévention du mouvement en réponse à une stimulation électrique supra maximale. ${ }^{4}$ Des rats knock-out pour le gène du récepteur de la mélanocortine-1 $(M C 1 R)$ ont également une concentration alvéolaire minimum (MAC, une mesure de la sensibilité à l'anesthésique basée sur le mouvement en réponse à la douleur supra-maximale) ${ }^{5}$ très légèrement supérieure aux rats de type sauvage. ${ }^{6}$ Chez la souris, les mutations MCIR agissent comme médiateurs de l'analgésie par les récepteurs kappa des opiacés, mais seulement chez les souris femelles. ${ }^{7}$ Au cours d'études ultérieures, les mêmes chercheurs ont trouvé que les mutations $M C 1 R$ chez les souris et les humains réduisent la sensibilité à la douleur et augmentent la réponse analgésique aux récepteurs $\mu$ des opiacés, ${ }^{8}$ et aussi que l'effet de ces mutations est dépendante du sexe chez les souris. ${ }^{9}$ En revanche, les cheveux roux n'influencent pas la pharmacodynamique du propofol. ${ }^{10}$

Bien que des récepteurs de la mélanocortine-1 aient été identifiés dans le tissu hypophysaire humain, dans les cellules gliales et dans les cellules de la substance grise périaqueducale $^{11,12}$ le système nerveux central n'est pas le principal site de l'expression de $M C 1 R{ }^{13}$ En outre, le MAC est probablement largement médié par la moelle épinière $^{14,15}$ plutôt que par des centres supérieurs. ${ }^{15,16} \mathrm{Il}$ existe néanmoins une interaction considérable entre les différents récepteurs de la mélanocortine ${ }^{17,18}$ car ils sont tous stimulés par la mélanocortine, l'hormone stimulant l' $\alpha$-mélanocortine (MSH), la $\beta$-MSH, la $\gamma$-MSH et l'hormone adrénocorticotrope (ACTH). Certains de ces récepteurs sont abondants dans le système nerveux central (SNC) et ont une affinité similaire pour l' $\alpha$-MSH et l'ACTH. ${ }^{13}$ Un antagonisme fonctionnel entre les systèmes opiacés et mélanocortine a été proposé, car les récepteurs sont retrouvés dans l'ensemble du SNC, y compris dans le locus cœruleus où leurs activités régulatrices s'opposent l'une à l'autre. ${ }^{19}$

Les roux sont également plus sensibles à la douleur thermique et résistants aux effets analgésiques de la lidocaïne sous-cutanée. ${ }^{20}$ Une étude transversale ultérieure a abouti à des conclusions compatibles avec ces résultats expérimentaux: les participants porteurs de variants du gène MC1R ont déclaré éprouver significativement plus d'anxiété envers les soins dentaires et de craintes de douleurs dentaires que les participants sans variant du gène MC1R. Les patients porteurs de la mutation étaient également deux fois plus susceptibles d'éviter les soins dentaires que les patients sans variants du gène $\mathrm{MC} 1 \mathrm{R}$, même après un ajustement pour le trait de personnalité anxieuse et le sexe. ${ }^{21}$

Dans ce numéro du Journal, Gradwohl et coll. ${ }^{22}$ décrivent une analyse secondaire de patients issus d'une étude publiée antérieurement sur l'éveil sous anesthésique chez des patients à risque élevé de souvenirs de l'évènement. ${ }^{23}$ Dans leur analyse, les investigateurs ont comparé quatre caractéristiques anesthésiques chez 319 patients roux autodéclarés et 1595 patients ayant d'autres couleurs de cheveux appariés sur les scores de propension.

Le premier critère d'évaluation étudié par les investigateurs était l'éveil avec souvenir. Même parmi les patients à haut risque qu'ils avaient recrutés, les auteurs s'attendaient à ce que seulement trois patients, environ, sur les 319 aient un souvenir de l'anesthésie; il n'y en eut que deux. Gradwohl et coll. n'ont donc pas une puissance statistique leur permettant de tirer une quelconque conclusion à partir de ces données sur le souvenir de la chirurgie.

Heureusement, Gradwohl et coll. sont bien mieux placés pour analyser la gestion anesthésique. Leurs résultats montrent, avec un niveau de confiance raisonnable, que l'administration d'anesthésiques volatils, d'adjuvants anesthésiques et d'opioïdes a été comparable chez les roux et chez les patients ayant d'autres couleurs de cheveux. Comme le notent les auteurs, les résultats indiquent au minimum que les anesthésiologistes des deux centres participants n'ont pas géré l'anesthésie de façon différente chez les roux par rapport aux patients non-roux.

Gradwohl et coll. ne confondent pas la quantité d'anesthésie administrée avec le besoin en anesthésique et il demeure possible que le besoin selon certaines mesures aurait en fait été différent chez les roux. Cette distinction est importante parce que les études précédentes sur les rongeurs et les humains ont évalué des mesures hautement spécifiques des besoins en anesthésiques, telles que le MAC et la sensibilité à la douleur dans des contextes optimaux non cliniques. Bien entendu, la difficulté réside dans le fait que l'anesthésie inclut la perte de connaissance, l'immobilité, le contrôle des réponses autonomes et l'amnésie. Il n'existe donc pas de mesure unitaire de l' "anesthésie », bien que l'indice bispectral de l'électroencéphalogramme est probablement la mesure globale unique la mieux acceptée et qu'il a été utilisé pour guider l'administration automatique de propofol et de remifentanil. ${ }^{24,25}$

Gradwohl et coll. ont ainsi utilisé la relation entre la pression partielle d'anesthésique volatil (MAC ajusté pour l'âge) et l'indice bispectral comme mesure de la sensibilité anesthésique, une technique que nous avons utilisée dans d'autres circonstances. ${ }^{26}$ L'analyse a été ajustée pour l'administration d'adjuvant anesthésique et d'opioïdes, ainsi que pour certaines caractéristiques propres aux patients et à la chirurgie. Il n'y a pas eu de différences cliniquement importantes dans la sensibilité aux anesthésiques entre les patients roux et les autres. Parmi les critères d'évaluation 
testés par Gradwohl et collaborateurs, la relation entre le MAC et l'indice bispectral est probablement la plus sensible pour les effets potentiels des cheveux roux sur l'anesthésie. Ils ont également évalué la durée de la récupération post anesthésie, ainsi que les nausées et vomissements. Aucune variation importante de ces critères n'était anticipée et, de fait, il n'y en a pas eu.

La question est alors de savoir pourquoi les résultats de Gradwohl et coll. diffèrent autant d'une demi-douzaine d'études exploratoires menées sur des rongeurs et chez des humains. L'explication la plus évidente est que les études précédentes évaluaient des critères d'aboutissements spécifiques, notamment le MAC et la sensibilité à la douleur dans des conditions optimales. De tels effets subtils peuvent être simplement indétectables, même dans une étude clinique soigneuse de cohorte. Mais il n'est pas inhabituel de voir plusieurs petites études cohérentes contredites par un grand essai ultérieur. ${ }^{27}$ En dehors du fait que les petites études sont statistiquement fragiles et peuvent donc être simplement fausses plus souvent qu'on ne le croit d'habitude, ${ }^{28}$ des détails méthodologiques fournissent parfois une explication.

Gradwohl et coll. ${ }^{22}$ ont été limités à des patients ayant eux-mêmes déclaré qu'ils avaient des cheveux naturellement roux. Leur étude diffère sur ce point de celle de Liem et coll. qui ont limité leur recrutement à des volontaires ayant des cheveux roux vif et qui ont effectué des tests biochimiques sur les cheveux ainsi qu'une analyse génétique chez leurs volontaires, et de l'étude de Binkley et coll. qui ont réalisé une étude génétique de leurs patients. ${ }^{21}$ Dans la mesure où Gradwohl et coll. ont inclus des patients qui n'auraient pas eu véritablement des cheveux naturellement roux (et la mutation prédisposante), cela diminuerait les différences apparentes avec leurs patients témoins.

L'étude de Gradwohl et coll. était une cohorte prospective plutôt qu'une étude randomisée contrôlée considérant qu'il est difficilement possible de randomiser la couleur des cheveux. Mais il en résulte que les investigateurs n'ont pas bénéficié de la protection contre les biais de sélection et les facteurs confondants que procure la randomisation ni de la protection contre les biais de mesures apportée par l'insu. La validité de leur analyse statistique est donc la clé de l'interprétation de leurs résultats, mais elle a été bien menée à l'aide des techniques appropriées.

En résumé, Gradwohl et coll. ${ }^{22}$ ont évalué différentes mesures de la sensibilité aux anesthésiques dans une cohorte prospective de patients. Ils ont trouvé que les roux et un groupe apparié de patients contrôles avaient reçu des quantités semblables d'anesthésiques volatils, d'opioïdes et d'adjuvants anesthésiques. En outre, la relation entre le MAC et l'indice bispectral a été comparable chez les roux et les patients ayant d'autres couleurs de cheveux. Leurs résultats suggèrent que les différences subtiles rapportées antérieurement sur la sensibilité aux anesthésiques volatils, aux anesthésiques locaux et aux opioïdes ne peuvent pas être extrapolées aux patients se déclarant spontanément roux.

Funded by internal sources only. The Department of Outcomes RESEARCH is funded by Covidien (Dublin, Ireland) which produces Bispectral Index monitors. The author does not have a personal financial interest related to this editorial.

Le financement a été assuré uniquement par des ressources internes. Le département Outcomes Research est financé par Covidien (Dublin, Irlande) qui produit les moniteurs d'indice bispectral. L'auteur n'a pas d'intérêt financier personnel en rapport avec cet éditorial.

Conflicts of interest None declared.

Conflits d'intérêts Aucun déclaré.

\section{References}

1. Rees JL, Flanagan N. Pigmentation, melanocortins and red hair. QJM 1999; 92: 125-31.

2. Schioth HB, Phillips SR, Rudzish R, Brich-Machin M, Wikberg $J E$, Rees JL. Loss of function mutations of the human melanocortin 1 receptor are common and are associated with red hair. Biochem Biophys Res Commun 1999; 260: 488-91.

3. Healy E, Jordan SA, Budd P, Suffolk R, Rees JL, Jackson IJ. Functional variation of MC1R alleles from red-haired individuals. Hum Mol Genet 2001; 10: 2397-402.

4. Liem EB, Lin CM, Suleman MI, et al. Anesthetic requirement is increased in redheads. Anesthesiology 2004; 101: 279-83.

5. Eger EI 2nd, Saidman LJ, Brandstater B. Minimum alveolar anesthetic concentration: a standard of anesthetic potency. Anesthesiology 1965; 26: 756-63.

6. Xing Y, Sonner JM, Eger EI 2nd, Cascio M, Sessler DI. Mice with a melanocortin 1 receptor mutation have a slightly greater minimum alveolar concentration than control mice. Anesthesiology 2004; 101: 544-6.

7. Mogil JS, Wilson SG, Chesler EJ, et al. The melanocortin-1 receptor gene mediates female-specific mechanisms of analgesia in mice and humans. Proc Natl Acad Sci U S A 2003; 100: 4867-72.

8. Mogil JS, Ritchie J, Smith SB, et al. Melanocortin-1 receptor gene variants affect pain and mu-opioid analgesia in mice and humans. J Med Genet 2005; 42: 583-7.

9. Juni A, Cai M, Stankova $M$, et al. Sex-specific mediation of opioid-induced hyperalgesia by the melanocortin-1 receptor. Anesthesiology 2010; 112: 181-8.

10. Doufas AG, Orhan-Sungur M, Komatsu R, et al. Bispectral index dynamics during propofol hypnosis is similar in red-haired and dark-haired subjects. Anesth Analg 2013; 116: 319-26.

11. Chhajlani V. Distribution of cDNA for melanocortin receptor subtypes in human tissues. Biochem Mol Biol Int 1996; 38: 73-80.

12. Wikberg JE. Melanocortin receptors: perspectives for novel drugs. Eur J Pharmacol 1999; 375: 295-310.

13. Abdel-Malek Z. Melanocortin receptors: their functions and regulation by physiological agonists and antagonists. Cell Mol Life Sci 2001; 58: 434-41.

14. Antognini J, Schwartz $K$. Exaggerated anesthetic requirements in the preferentially anesthetized brain. Anesthesiology 1993; 79: 1244-9. 
15. Rampil IJ, Mason P, Singh H. Anesthetic potency (MAC) is independent of forebrain structures in the rat. Anesthesiology 1993; 78: 707-12.

16. Rampil IJ. Anesthetic potency is not altered after hypothermic spinal cord transection in rats. Anesthesiology 1994; 80: 606-10.

17. Cone RD, Mountjoy $K G$, Robbins LS, et al. Cloning and functional characterization of a family of receptors for the melanotropic peptides. Ann N Y Acad Sci 1993; 31: 342-63.

18. Mountjoy KG, Robbins LS, Mortrud MT, Cone RD. The cloning of a family of genes that encode the melanocortin receptors. Science 1992; 28: 1248-51.

19. Rene F, Muller A, Jover E, Kieffer B, Koch B, Loeffler JP. Melanocortin receptors and delta-opioid receptor mediate opposite signalling actions of POMC-derived peptides in CATH.a cells. Eur J Neurosci 1998; 10: 1885-94.

20. Liem EB, Joiner TV, Tsueda K, Sessler DI. Increased sensitivity to thermal pain and reduced subcutaneous lidocaine efficacy in redheads. Anesthesiology 2005; 102: 509-14.

21. Binkley CJ, Beacham A, Neace W, Gregg RG, Liem EB, Sessler $D I$. Genetic variations associated with red hair color and fear of dental pain, anxiety regarding dental care and avoidance of dental care. J Am Dent Assoc 2009; 140: 896-905.
22. Gradwohl SC, Aranake A, Abdallah AB, et al. Intraoperative awareness risk, anesthetic sensitivity, and anesthetic management for patients with natural red hair: a matched cohort study, in press. Can J Anesth 2015; 62: this issue; DOI: 10.1007/s12630014-0305-8.

23. Avidan MS, Jacobsohn E, Glick D, et al. Prevention of intraoperative awareness in a high-risk surgical population. N Engl J Med 2011; 365: 591-600.

24. Le Guen M, Liu N, Bourgeois E, et al. Automated sedation outperforms manual administration of propofol and remifentanil in critically ill patients with deep sedation: a randomized phase II trial. Intensive Care Med 2013; 39: 454-62.

25. Liu N, Chazot T, Hamada S, et al. Closed-loop coadministration of propofol and remifentanil guided by bispectral index: a randomized multicenter study. Anesth Analg 2011; 112: 546-57.

26. Perez-Protto S, Geube M, Ontaneda D, Dalton JE, Kurz A, Sessler DI. Sensitivity to volatile anesthetics in patients with dementia: a case-control analysis. Can J Anesth 2014; 61: 611-8.

27. Ioannidis $J P$. Contradicted and initially stronger effects in highly cited clinical research. JAMA 2005; 294: 218-28.

28. Ioannidis $J P$. Why most published research findings are false. PLoS Med 2005; 2: e124. 